\title{
EFECTO DE CIHALOFOP-BUTILO EN EL CONTROL DE MALEZAS GRAMÍNEAS ANUALES EN ARROZ DE TEMPORAL ${ }^{1}$
}

\author{
Valentín A. Esqueda², Oscar H. Tosquy ${ }^{2}$
}

\begin{abstract}
RESUMEN
Efecto de cihalofop-butilo para el control de malezas gramíneas anuales en arroz de temporal. Con objeto de evaluar la efectividad del herbicida cihalofop-butilo en el control de malezas gramíneas anuales en el arroz de temporal cv Milagro Filipino, durante 2002, se condujeron dos experimentos en el municipio de Tres Valles, Ver., México. En ambos experimentos se utilizó el diseño experimental de bloques al azar con siete tratamientos y cuatro repeticiones: cihalofop-butilo a $180,225,270,315$ y $360 \mathrm{~g} / \mathrm{ha}$, propanil a $4.320 \mathrm{~g} / \mathrm{ha}$ y un testigo sin aplicar. Los tratamientos se aplicaron cuando el arroz tenía una altura de aproximadamente $25 \mathrm{~cm}$. Las malezas gramíneas presentes fueron el zacate de agua y el zacate frente de toro [Digitaria ciliaris (Retz.) Koeler]. El control de malezas gramíneas anuales y la toxicidad del herbicida al arroz se evaluaron a los 15, 30 y 45 días después de la aplicación. Los mejores controles de los zacates de agua y frente de toro se obtuvieron cuando cihalofop-butilo se aplicó a 315 y 360 g/ha. Las diferentes dosis de este herbicida, no ocasionaron toxicidad al arroz. El efecto en el control de las malezas gramíneas por el propanil fue similar al de cihalofop-butilo en dosis de $360 \mathrm{~g} / \mathrm{ha}$ durante los primeros 15 días después de su aplicación.
\end{abstract}

Palabras claves: malezas gramíneas, arroz de temporal, cihalofop-butilo, control químico, Echinochola colona, Digitaria ciliaris.

\begin{abstract}
Effect of cyhalofop-butyl for annual grass weed control in rain-fed rice. In 2002, two experiments were carried out in the municipality of Tres Valles, Ver., Mexico, in order to evaluate the effectiveness of cyhalofop-butyl on the annual grass weed control in rain-fed rice cv Milagro Filipino. A randomized complete block design with seven treatments and four replications was utilized for both experiments. Cyhalofop-butyl was evaluated at 180, 225, 270,315 y $360 \mathrm{~g} / \mathrm{ha}$, propanil at $4320 \mathrm{~g} / \mathrm{ha}$ and a weedy check were also included. The treatments were applied when the rice plants had a height of $25 \mathrm{~cm}$. Annual grass weeds present in the experiments, were junglerice and Southern crabgrass [Digitaria ciliaris (Retz.) Koeler]. Grass weed control and toxicity to rice were evaluated at 15,30 and 45 days after application. The highest junglerice and Southern crabgrass controls were obtained when cyhalofop-butyl was applied at 315 and $360 \mathrm{~g} / \mathrm{ha}$. The different dosages of this herbicide, did not cause any toxicity to rice. The effect of propanil on weeds was similar to that of cyhalofop-butyl at $360 \mathrm{~g} / \mathrm{ha}$ during the first 15 days after application.
\end{abstract}

Key words: grass weeds, rainfed rice, cyhalofop-butyl, chemical control, Echinochloa colona, Digitaria ciliaris.

\section{INTRODUCCIÓN}

Las condiciones de alta humedad y temperatura en que se produce el arroz de temporal, son propicias para la presencia y el desarrollo de grandes poblaciones de malezas, que si no son controladas oportuna y eficientemente, pueden reducir de 30 a $50 \%$ el rendimiento de grano, y en casos extremos ocasionar la pérdida total de la cosecha (Esqueda 1990). Las malezas más importantes en el cultivo de arroz de temporal, son las gramíneas anuales (conocidas regionalmente como zacates), dentro de las cuales, la principal especie es el zacate de agua, también conocido como zacate pinto o zacate colorado [Echinochloa colona (L.) Link], que puede presentarse en poblaciones superiores a los 10000.000 de plantas/ha (Esqueda 2000b, Esqueda y Rosales 2004).

1 Recibido para publicación el 11 de junio del 2004.

2 Campo Experimental Cotaxtla. CIRGOC. INIFAP. SAGARPA. esqueda.valentin@inifap.gob.mx 
En el estado de Veracruz, los herbicidas comerciales más utilizados para el control de los zacates anuales, están formulados con propanil, un producto del grupo químico de las amidas, desarrollado a principios de los 60’s, que se aplica en postemergencia (Smith 1961, Thomson 1993, Fontanilla et al. 2001). El propanil actúa mejor cuando los zacates son pequeños y están creciendo activamente y su control es deficiente cuando se aplica a zacates de gran tamaño o que se encuentran en la etapa de amacollamiento (Leah et al. 1995). Debido a que el propanil no es un herbicida residual, normalmente se requiere de más de una aplicación en el ciclo de vida del arroz, o bien mezclarlo con un herbicida preemergente (Stauber et al. 1991, Esqueda 2000a, Ntanos et al. 2000). En los últimos años, el abuso en el número de aplicaciones y el empleo de dosis elevadas, han ocasionado la aparición de biotipos de zacate de agua, que presentan varios grados de resistencia al propanil en América Central y América del Sur (Garro et al. 1993, Fischer et al. 1993, Ortiz et al. 1999). En México, ya se han identificado poblaciones de zacate de agua tolerantes al propanil, en arrozales de los estados de Veracruz y Campeche (Bolaños et al. 2001).

El cihalofop-butilo es un herbicida desarrollado para el control de gramíneas en el cultivo de arroz (Ito et al. 1998, Mann et al. 2000). Una de las ventajas que presenta con respecto al propanil, es que puede ser utilizado para el control de zacates de mayor tamaño que los que controla este herbicida. Además, como tiene un modo de acción diferente al de propanil, representa una alternativa para evitar o retrasar la aparición de biotipos de zacates resistentes a este herbicida o para controlarlos, si es que ya están presentes (Caseley et al. 1997, Valverde et al. 2000). Debido a que se pretende comercializar el cihalofop-butilo en México, es necesario conocer el comportamiento de este herbicida sobre las poblaciones de zacate de agua y otras malezas gramíneas, en las condiciones agroclimáticas en que se produce el arroz de temporal en nuestro país. Por lo anterior, se establecieron dos experimentos con el objetivo de determinar el efecto del cihalofop-butilo en el control de malezas gramíneas anuales en el cultivo de arroz de temporal y su selectividad a la variedad de arroz Milagro Filipino de uso generalizado en el estado de Veracruz.

\section{MATERIALES Y MÉTODOS}

Los experimentos se establecieron durante el ciclo de lluvias de 2002, en Mata Verde (experimento 1) y la Col. Independencia (experimento 2), dos localidades arroceras del municipio de Tres Valles, Veracruz, México. Se utilizó semilla de arroz de la variedad Milagro Filipino, sembrada "a chorrillo" a una densidad de 100 $\mathrm{kg} / \mathrm{ha}$. En ambos experimentos se evaluaron siete tratamientos, entre los que se incluyeron un testigo sin aplicar y el herbicida propanil como testigo regional. Los tratamientos se aplicaron a los 16 y 18 días después de la emergencia del arroz en Col. Independencia y Mata verde, respectivamente (Cuadro 1).

Cuadro 1. Tratamientos y época de aplicación por localidad de evaluación. Tres Valles, Veracruz, México. 2002.

\begin{tabular}{clc}
\hline No. & Tratamiento* $^{*}$ & Dosis (g i. a./ha) \\
\hline 1 & Cihalofop-butilo & 180 \\
2 & Cihalofop-butilo & 225 \\
3 & Cihalofop-butilo & 270 \\
4 & Cihalofop-butilo & 315 \\
5 & Cihalofop-butilo & 360 \\
6 & Propanil ** & 4.320 \\
7 & Testigo sin aplicar & - \\
\hline
\end{tabular}

* A los tratamientos del 1 al 6 se les adicionó un surfactante no iónico en dosis de $250 \mathrm{ml}$, por cada $100 \mathrm{l}$ de agua.

** Testigo regional

Se utilizó el diseño experimental de bloques completos al azar con cuatro repeticiones. Cada parcela experimental estuvo constituida por diez surcos de $10 \mathrm{~m}$ de longitud, espaciados a $0,30 \mathrm{~m}$, equivalente a una superficie de $30 \mathrm{~m}^{2}$.

En el experimento 1, los tratamientos se aplicaron cuando el arroz tenía entre cinco y seis hojas y una altura de entre 22 y $30 \mathrm{~cm}$. Al momento de la aplicación, las malezas gramíneas tenían entre 10 y 14 hojas y una altura de entre 22 y $35 \mathrm{~cm}$. En el experimento 2, la aplicación de los tratamientos se llevó a cabo cuando el arroz tenía entre cuatro y cinco hojas y una altura de entre 20 y $30 \mathrm{~cm}$ y las malezas gramíneas tenían entre siete y 16 hojas y una altura de entre 20 y $48 \mathrm{~cm}$. Los tratamientos se aplicaron solamente en los ocho surcos centrales, para utilizar los surcos de las orillas como testigos laterales enhierbados. Debido a que cihalofopbutilo es un herbicida específico para controlar malezas gramíneas, a los $25 \mathrm{DDE}$ en todas las parcelas tratadas con herbicidas, se aplicó 2,4-D amina a $479 \mathrm{~g} / \mathrm{ha}$ para controlar las malezas de hoja ancha.

La densidad de población de malezas se determinó al momento de la aplicación de los tratamientos. Para esto, se utilizó un cuadrante de 0,5 x $0,5 \mathrm{~m}$, el cual fue lanzado al azar en las parcelas correspondientes a los testigos sin aplicar. Las malezas del interior de los cuadrantes fueron identificadas y cuantificadas por especie. Las evaluaciones de control de malezas se llevaron a cabo a los 15, 30 y 45 días después de la aplicación de 
los tratamientos (DDA). Se evaluó visualmente el efecto de los herbicidas sobre las especies de malezas dominantes en la totalidad de cada parcela experimental. Para ello, se utilizó la escala porcentual (0 - 100\%), en donde 0 , significó que las malezas no fueron afectadas y 100, que fueron completamente eliminadas (Ntanos et al. 2000, Esqueda y Rosales 2004).

La selectividad de los diferentes herbicidas y dosis al arroz, se evaluó en las mismas fechas que el control de las malezas; las evaluaciones se llevaron a cabo de forma visual y se asignaron valores en la escala de 0 a $100 \%$, en donde 0 , significó que el arroz no fue afectado y 100 , que fue completamente destruido.

Los datos experimentales de porcentaje de control de malezas fueron transformados a su valor de arco seno $\sqrt{\%}$, como se recomienda en Gómez y Gómez (1984). Los análisis de varianza se efectuaron con los datos transformados y como prueba de separación de medias se utilizó Tukey al 0,05. Aún cuando los análisis realizados se efectuaron con datos transformados, para dar mayor claridad a los resultados, se presentan los valores reales.

\section{RESULTADOS Y DISCUSIÓN}

Experimento 1 (Mata Verde). En el sitio experimental se presentaron siete especies de malezas de ciclo de vida anual, pertenecientes a cinco familias botánicas, cuya densidad de población total fue de 10020.000 plantas/ha (Cuadro 2). Las malezas gramíneas presentes, fueron los zacates de agua y frente de toro [Digitaria ciliaris (Retz.) Koeler], cuyas poblaciones fueron de 2740.000 y 1610.000 plantas/ha, respectivamente.
A los 15 DDA, los mejores controles de E. colona, se obtuvieron con propanil, así como con las dosis de 315 y $360 \mathrm{~g} / \mathrm{ha}$ de cihalofop-butilo, siendo estadísticamente semejantes entre sí. A su vez, se observó, que el control de esta especie disminuyó conforme se redujeron las dosis de cihalofop-butilo. De esta manera, mientras que con la dosis de $180 \mathrm{~g} / \mathrm{ha}$ se obtuvo un control de $59 \%$, con la de $360 \mathrm{~g} / \mathrm{ha}$, el control fue de $88 \%$. A los 30 DDA, se observó un ligero incremento (entre 2 y $6 \%$ ) en el control de E. colona en todos los tratamientos de cihalofop-butilo; en esta época de evaluación, los controles más altos se obtuvieron con las dosis de 360 , 315 y $270 \mathrm{~g} / \mathrm{ha}$, y fueron estadísticamente semejantes entre sí. Por otra parte, con propanil se redujo significativamente el control de esta especie, aunque éste fue estadísticamente similar a los controles obtenidos con cihalofop-butilo, a 180, 225 y $270 \mathrm{~g} / \mathrm{ha}$. A los 45 DDA, los controles de E. colona tuvieron pocos cambios respecto a los observados en la evaluación anterior, manteniéndose cihalofop-butilo a 360 y $315 \mathrm{~g} / \mathrm{ha}$, como los mejores tratamientos, con 91 y $88 \%$ de control, respectivamente (Cuadro 3).

Cuadro 3. Efecto de los tratamientos, en el control de Echinochloa colona (\%) a los 15, 30 y 45 DDA. Mata Verde, Tres Valles, Veracruz, México. 2002.

\begin{tabular}{lcccc}
\hline \multicolumn{1}{c}{ Tratamiento } & 15 DDA & 30 DDA & 45 DDA \\
\hline 1. Cihalofop-butilo (180 g/ha) & $59 \mathrm{~d}^{*}$ & $63 \mathrm{c}$ & $59 \mathrm{~d}$ \\
2. Cihalofop-butilo (225 g/ha) & $68 \mathrm{~cd}$ & $70 \mathrm{bc}$ & $70 \mathrm{~cd}$ \\
3. Cihalofop-butilo (270 g/ha) & $75 \mathrm{bc}$ & $81 \mathrm{ab}$ & $79 \mathrm{bc}$ \\
4. Cihalofop-butilo (315 g/ha) & $80 \mathrm{ab}$ & $86 \mathrm{a}$ & $88 \mathrm{ab}$ \\
5. Cihalofop-butilo (360 g/ha) & $88 \mathrm{a}$ & $91 \mathrm{a}$ & $91 \mathrm{a}$ \\
6. Propanil (4.320 g/ha) & $88 \mathrm{a}$ & $74 \mathrm{bc}$ & $71 \mathrm{~cd}$ \\
7. Testigo sin aplicar & 0 & 0 & 0 \\
\hline
\end{tabular}

$*$ Tukey $\leq 0,05$.

Cuadro 2. Especies y poblaciones de malezas presentes en el experimento ubicados en Mata Verde. Mata Verde, Tres Valles, Veracruz, México. 2002.

\begin{tabular}{llllr}
\hline Nombre común & \multicolumn{1}{c}{ Nombre científico } & Familia* & Ciclo de vida & $\begin{array}{r}\text { Densidad } \\
\text { plantas/ha }\end{array}$ \\
\hline Pelo de conejo & Cyperus iria L. & Cyperaceae & Anual & 5360.000 \\
Z. de agua & Echinochloa colona (L.) Link & Poaceae & Anual & 2740.000 \\
Z. frente de toro & Digitaria ciliaris (Retz.) Koeler & Poaceae & Anual & 1610.000 \\
Palmilla & Aeschynomene americana L. & Fabaceae & Anual & 180.000 \\
Tabaquillo & Richardia scabra L. & Rubiaceae & Anual & 80.000 \\
Papayita & Croton lobatus L. & Euphorbiaceae & Anual & 40.000 \\
Pantanillo & Caperonia palustris (L.) St. Hil. & Euphorbiaceae & Anual & 10.000 \\
Total & & & & 10020.000 \\
\hline
\end{tabular}

* Se utilizó el nombre de la familia botánica indicado por Villaseñor y Espinosa (1998). 
Con respecto a $D$. ciliaris (Cuadro 4), a los 15 DDA, el control más alto (91\%), se obtuvo al aplicar propanil, el cual fue estadísticamente semejante a los tratamientos de cihalofop-butilo, en dosis de 270,315 y $360 \mathrm{~g} / \mathrm{ha}$, cuyos controles fueron de 84,86 y $89 \%$, respectivamente. A su vez, los controles de esta especie con las dosis de 180 y $225 \mathrm{~g} / \mathrm{ha}$, fueron de solamente 59 y $66 \%$. A los 30 DDA, en la mayoría de los tratamientos donde se aplicó el cihalofop-butilo, se observó un incremento en el control de $D$. ciliaris, aunque éste fue menor al observado en E. colona. En esta época, los controles más altos se observaron en las parcelas en donde el cihalofop-butilo se aplicó a 315 y $360 \mathrm{~g} / \mathrm{ha}$. Por otra parte, con propanil, se tuvo un control de esta especie, similar al obtenido con cihalofop-butilo a 270 y $315 \mathrm{~g} / \mathrm{ha}$, mientras que con las dosis de 180 y 225 $\mathrm{g} /$ ha se obtuvieron los controles más bajos. En la última época de evaluación, los controles de $D$. ciliaris tuvieron pocos cambios con respecto a la evaluación anterior, y los controles más altos (93 y 90\%), se obtuvieron con las dosis de 360 y $315 \mathrm{~g} /$ ha de cihalofop-butilo (Cuadro 4).

Cuadro 4. Efecto de los tratamientos en el control de Digitaria ciliaris (\%) a los 15, 30 y 45 DDA. Mata Verde, Tres Valles, Veracruz. 2002.

\begin{tabular}{lcrl}
\hline \multicolumn{1}{c}{ Tratamiento } & 15 DDA & 30 DDA & 45 DDA \\
\hline 1. Cihalofop-butilo $(180 \mathrm{~g} / \mathrm{ha})$ & $59 \mathrm{~b}^{*}$ & $61 \mathrm{c}$ & $60 \mathrm{~d}$ \\
2. Cihalofop-butilo $(225 \mathrm{~g} / \mathrm{ha})$ & $66 \mathrm{~b}$ & $68 \mathrm{c}$ & $70 \mathrm{~cd}$ \\
3. Cihalofop-butilo $(270 \mathrm{~g} / \mathrm{ha})$ & $84 \mathrm{a}$ & $83 \mathrm{~b}$ & $80 \mathrm{bc}$ \\
4. Cihalofop-butilo $(315 \mathrm{~g} / \mathrm{ha})$ & $86 \mathrm{a}$ & $88 \mathrm{ab}$ & $90 \mathrm{ab}$ \\
5. Cihalofop-butilo $(360 \mathrm{~g} / \mathrm{ha})$ & $89 \mathrm{a}$ & $91 \mathrm{a}$ & $93 \mathrm{a}$ \\
6. Propanil $(4320 \mathrm{~g} / \mathrm{ha})$ & $91 \mathrm{a}$ & $83 \mathrm{~b}$ & $80 \mathrm{bc}$ \\
7. Testigo sin aplicar & 0 & 0 & 0 \\
\hline
\end{tabular}

$*$ Tukey $\leq 0,05$.
Durante las evaluaciones realizadas, ni el propanil, ni ninguna de las dosis de cihalofop-butilo ocasionó toxicidad a la variedad de arroz Milagro Filipino.

Experimento 2 (Col. Independencia). En este lote experimental, se presentaron siete especies de malezas, pertenecientes a cuatro familias botánicas. Seis especies de ciclo de vida anual y una perenne. Al momento de la aplicación de los herbicidas, la población de malezas era de 5050.000 plantas/ha, siendo E. colona la especie gramínea dominante, con una población de 800.000 plantas/ha (Cuadro 5).

A los 15 DDA, el control más alto de E. colona $(86 \%)$, se obtuvo con la aplicación de propanil, el cual fue estadísticamente semejante al control de $83 \%$, obtenido cuando se aplicó cihalofop-butilo a $360 \mathrm{~g} / \mathrm{ha}$. La efectividad del cihalofop-butilo sobre E. colona, fue directamente proporcional a la dosis aplicada; de esta manera, mientras que con la dosis de $315 \mathrm{~g} /$ ha se obtuvo un control de $76 \%$, con la dosis de $180 \mathrm{~g} / \mathrm{ha}$, el control fue de solamente $43 \%$. A los 30 DDA se observó un ligero incremento en el control de E. colona en los tratamientos de cihalofop-butilo a las dosis de 180, 225 y $270 \mathrm{~g} / \mathrm{ha}$, mientras que a las dosis de 315 y $360 \mathrm{~g} / \mathrm{ha}$, el control de esta especie prácticamente se mantuvo igual que a los 15 DDA. En esta época de evaluación, el mayor control se obtuvo con cihalofop-butilo a 360 $\mathrm{g} / \mathrm{ha}$, aunque estadísticamente fue semejante a los controles obtenidos con las dosis de 270 y $315 \mathrm{~g} / \mathrm{ha}$ y con propanil a $4.320 \mathrm{~g} / \mathrm{ha}$. En la última época de evaluación, la aplicación de cihalofop-butilo a $360 \mathrm{~g} / \mathrm{ha}$ tuvo un control de E. colona significativamente superior al obtenido por propanil y fue estadísticamente semejante a los controles que se tuvieron con las dosis de $270 \mathrm{y}$ $315 \mathrm{~g} / \mathrm{ha}$ del mismo herbicida (Cuadro 6).

Cuadro 5. Especies y poblaciones de malezas presentes en el experimento de la Colonia Independencia. Col. Independencia, Tres Valles, Veracruz, México. 2002.

\begin{tabular}{llllr}
\hline Nombre común & \multicolumn{1}{c}{ Nombre científico } & Familia* & Ciclo de vida & $\begin{array}{c}\text { Densidad } \\
\text { plantas/ha }\end{array}$ \\
\cline { 2 - 4 } & & & Anual & 3890.000 \\
Pelo de conejo & Cyperus iria L. & Cyperaceae & Anual & 800.000 \\
Z. de agua & Echinochloa colona (L.) Link & Poaceae & Anual & 130.000 \\
Collarcillo & Phyllanthus niruri L. & Euphorbiaceae & Anual & 90.000 \\
Papayita & Croton lobatus L. & Euphorbiaceae & Anual & 70.000 \\
Chilillo & Polanisia viscosa (L.) DC. & Capparaceae & Anual & 50.000 \\
Navajuela & Scleria sp. & Cyperaceae & Anual & 20.000 \\
Zacate Johnson & Sorghum halepense (L.) Pers. & Poaceae & Perenne & 5050.000 \\
Total & & & & \\
\hline
\end{tabular}

* Se utilizó el nombre de la familia botánica indicado por Villaseñor y Espinosa (1998). 
Cuadro 6. Efecto de los tratamientos, en el control de Echinochloa colona (\%) a los 15, 30 y 45 DDA. Col. Independencia, Tres Valles, Veracruz, México. 2002.

\begin{tabular}{lcccc}
\hline \multicolumn{1}{c}{ Tratamiento } & 15 DDA & 30 DDA & 45 DDA \\
\hline 1. Cihalofop-butilo $(180 \mathrm{~g} / \mathrm{ha})$ & $43 \mathrm{e} *$ & $55 \mathrm{c}$ & $50 \mathrm{c}$ \\
2. Cihalofop-butilo $(225 \mathrm{~g} / \mathrm{ha})$ & $59 \mathrm{~d}$ & $66 \mathrm{bc}$ & $68 \mathrm{~b}$ \\
3. Cihalofop-butilo $(270 \mathrm{~g} / \mathrm{ha})$ & $68 \mathrm{~cd}$ & $74 \mathrm{ab}$ & $75 \mathrm{ab}$ \\
4. Cihalofop-butilo $(315 \mathrm{~g} / \mathrm{ha})$ & $76 \mathrm{bc}$ & $75 \mathrm{ab}$ & $80 \mathrm{ab}$ \\
5. Cihalofop-butilo $(360 \mathrm{~g} / \mathrm{ha})$ & $83 \mathrm{ab}$ & $83 \mathrm{a}$ & $84 \mathrm{a}$ \\
6. Propanil (4320 g/ha) & $86 \mathrm{a}$ & $73 \mathrm{ab}$ & $69 \mathrm{~b}$ \\
7. Testigo sin aplicar & $0 \mathrm{f}$ & $0 \mathrm{~d}$ & $0 \mathrm{~d}$ \\
\hline
\end{tabular}

* Tukey 0,05 .

No se observaron síntomas de toxicidad en las plantas de arroz, con ninguno de los tratamientos evaluados.

Del análisis de los resultados, se considera que el cihalofop-butilo, puede utilizarse para el control de $E$. colona y $D$. ciliaris en arroz de temporal, a partir de la dosis de $315 \mathrm{~g} / \mathrm{ha}$. Es posible que al aplicar cihalofopbutilo a plantas de menor tamaño, la dosis de este herbicida pueda reducirse, ya que Ntanos et al. (2000) encontraron que el cihalofop-butilo a las dosis de $150 \mathrm{y}$ $200 \mathrm{~g} / \mathrm{ha}$, controló eficientemente plántulas de E. crusgalli (una especie presente en las áreas subtropicales), cuando éstas tenían dos y cuatro hojas. En apoyo a lo anterior, en Mata Verde, a pesar de tener una mayor densidad de población, los controles de E. colona fueron ligeramente superiores a los de la Col. Independencia, en donde la altura promedio de las plantas fue mayor. Es importante recalcar, que al ser cihalofop-butilo un herbicida exclusivo para el control de malezas gramíneas, es necesario utilizar posteriormente otro herbicida para controlar las malezas de hoja ancha.

Un control eficiente de E. colona y D. ciliaris se obtuvo a partir de la dosis de $315 \mathrm{~g} / \mathrm{ha}$ de cihalofop-butilo.

El herbicida cihalofop-butilo fue altamente selectivo a la variedad de arroz Milagro Filipino.

El propanil solamente tuvo un efecto semejante en el control de las gramíneas anuales a la dosis mayor de cihalofop-butilo, durante los primeros 15 días después de la aplicación.

Cihalofop-butilo representó una alternativa de uso para evitar o retrasar la aparición de biotipos de $E$. colona resistente al propanil.

\section{LITERATURA CITADA}

BOLAÑOS, E.; VILLA, J. T.; VALVERDE, B. E. 2001. Respuesta de Echinochloa colona (L.) Link a propanil en áreas arroceras selectas de México. Revista Mexicana de la Ciencia de la Maleza. 1:21-26.

CASELEY, J. C.; PALGRAVE, C.; HAAS, E. 1997. Herbicides with alternative modes of action for the control of propanil- and fenoxaprop-p-resistant Echinochloa colona. Proc. Brighton Crop Prot. Conf. 3:215-220.

ESQUEDA, V. A. 1990. La maleza y su control en arroz de temporal en México. Series Técnicas de ASOMECIMA 1:12-16

ESQUEDA, V. A. 2000a. Control de malezas en arroz de temporal con clomazone, solo y en mezcla con propanil y 2,4-D. Agronomía Mesoamericana 11:51-56.

ESQUEDA, V. A. 2000b. Las malezas del cultivo del arroz (Oryza sativa L.) en México. Revista Mexicana de la Ciencia de la Maleza. Núm. Especial. p. 63-81.

ESQUEDA, V. A.; ROSALES, E. 2004. Evaluación de bispiribac-sodio en el control de malezas en arroz de temporal. Agronomía Mesoamericana 15(1):9-15.

FISCHER, A. J.; GRANADOS, E.; TRUJILLO, D. 1993. Propanil resistance in populations of junglerice (Echinochloa colona) in Colombian rice fields. Weed Sci. 41:201-206.

FONTANILLA, J. M.; RUIZ-SANTAELLA, J. P.; GARRIDO, R.; DE PRADO, R. 2001. Efecto de diferentes formulaciones de propanil sobre arroz (var. Bahía) y Echinochloa crus-galli. In: R. De Prado y J. V. Jorrín (eds.). Uso de herbicidas en la agricultura del Siglo XXI. Córdoba; Servicio de Publicaciones de la Universidad de Córdoba. p. 433-439.

GARRO, J. E.; DE LA CRUZ, R.; MERAYO, A. 1993. Estudio del crecimiento de materiales de Echinochloa colo$n a$ (L.) Link. susceptibles y tolerantes al propanil. Manejo Integrado de Plagas 26:39-43.

GOMEZ, K. A.; GOMEZ, A. A. 1984. Statistical Procedures for Agricultural Research. 2nd. ed. New York, J. Wiley \& Sons. $680 \mathrm{p}$.

ITO, M.; KAWAHARA, H.; ASAI, M. 1998. Selectivity of cyhalofop-butyl in Poaceae species. J. Weed Sci. and Technol. 43:122-128. 
LEAH, J. M.; CASELEY, J. C.; RICHES, C. R; VALVERDE, B. E. 1995. Age-related mechanisms of propanil tolerance in jungle-rice, Echinochloa colona. Pesticide Sci. 43:347-354.

MANN, R.; LASSITER, R.; SIMPSON, D.; GRANT, D.; RICHBURG, J.; LANGSTON, V. 2000. Cyhalofop, a new postemergence graminicide in rice. In: Proceedings Southern Weed Science Society 53rd Annual Meeting. Y2K: a challenge for a change. p. 169-170.

NTANOS, D. A.; KOUTROUBAS, S. D.; MAVROTAS, C. 2000. Barnyardgrass (Echinochloa crus-galli) control in water-seeded rice (Oryza sativa) with cyhalofopbutyl. Weed Technol. 14:383-388.

ORTÍZ, A.; PACHECO, M.; PÉREZ, V; RAMOS, R.; SEJÍAS, E. 1999. Identificación de biotipos de Echinochloa colona (L.) Link, potencialmente resistentes al propanil en Venezuela. Rev. COMALFI 26:21-27.

SMITH, R. J. JR. 1961. 3,4-dichloropropionanilide for control of barnyardgrass in rice. Weeds 3:318-322.
STAUBER, L. G.; NASTASI, P.; SMITH JR., R.; BALTAZAR, A.; TALBERT, R. 1991. Barnyardgrass (Echinochloa crus-galli) and sprangletop (Leptochloa fascicularis) control in rice (Oryza sativa). Weed Technol. 5(2):337-344.

THOMSON, W. T. 1993. Agricultural Chemicals. Book II Herbicides. Fresno, CA, Thomson Publications. 310 p.

VALVERDE, B. E.; RICHES, C. R.; CASELEY, J. C. 2000 Prevención y manejo de malezas resistentes a herbicidas en arroz: experiencias en América Central con Echinochloa colona. Traducción del inglés por Bernal E. Valverde. Cámara de Insumos Agropecuarios. San José, Costa Rica. 135 p.

VILLASEÑOR, J. L.; ESPINOSA, F. J. 1998. Catálogo de malezas de México. México. Universidad Nacional Autónoma de México. Consejo Nacional Consultivo Fitosanitario. Fondo de Cultura Económica. 449 p. 